\title{
Substituent Effect in Sensitization Mechanisms of Pyrromethene Dyes in Solution
}

\author{
Toshiyuki Urano, ${ }^{* a(b)}$ Eiko Ohno-Okumurac), Keiichi Sakamoto ${ }^{d)}$, Shota Suzuki ${ }^{\text {e) }}$, \\ Tsuguo Yamaoka ${ }^{e}$, Naoto Hara ${ }^{n}$, Keijiro Fukui ${ }^{n}$, Takashi Karatsu ${ }^{n}$, and \\ Akihide Kitamuran \\ a)Research Center, Mitsubishi Kagaku Corporation, Aoba-ku, Yokohama 227 Japan \\ b) Center for cooperative research, Chiba University Inage-ku, Chiba 263-8522, \\ Japan \\ b) Research Institute of Chemical Science, Technology and Education, 8-37-104 \\ Narashinodai, Funabashi, Chiba 274-0063 Japan \\ c) Department of Industrial Chemistry, College of Industrial Technology, Nihon \\ University, 1-2-1 Izumi, Narashino, Chiba 275-8575 Japan \\ e) Department of Information and Image Science, Faculty of Engineering \\ Chiba University, Inage-ku, Chiba 263-8522, Japan \\ f) Department of Materials Technology, Faculty of Engineering, Chiba \\ University, Inage-ku, Chiba 263-8522, Japan.
}

The sensitization mechanisms for photo-initiations comprised a sensitizer dyes such as 2,8 diethyl-1,3,5,7,9-pentamethylbipyrromethene difluoroborate (EBP), 1,3,5,7,9-pentamethylbipyrromethene difluoroborate (HBP), and 2,8-diethyl-1,3,5,7-teramethyl-9-phenylbipyrromethene difluoroborate (PBP), and a radical generating reagent 3,3',4,4'-tetrakis(tbutylperoxycarbonyl) benzophenone (BTTB) in acetonitrile have been investigated by singe photon counting.

KEYWORDS: Sensitizer, pyrromethene, polymerization, liquid photoresist.

\section{Introduction}

Sensitization mechanisms by pyrromethene dyes were investigated by single photon counting. In the sensitized decomposition of 3,3',4,4'-tetrakis(t-butylperoxycarbonyl) benzophenone (BTTB), Fouassier reported that the sensitization mechanisms of photoinitiating system comprised of a sensitizing dye, thiopyrylium salt (TP), and BTTB, in which a triplet sensitization process was predominat with the triplet-quenching rate constant $\mathrm{kq}=6.6$ x $10^{7} \mathrm{~mol}^{-1} \mathrm{dm}^{3} \mathrm{~s}^{-1}$, and a singlet-excited sensitization process was minor due to rather high triplet quantum yield $\left(\Phi_{T}\right), \Phi_{T}=0.3[1]$ and low fluorescent character [2]. In the contrast to the mechanisms between $\mathrm{TP}$ and pyrromethene dyes, the pyrromethene dyes are well known as highly efficient fluorescent dyes with fluorescence quantum yield $\left(\Phi_{i}\right), \Phi_{f}>0.8$, which can be applied to laser dyes [3, 4], fluorescent probes in biochemical procedure [5], and sensitizer dyes of solid and liquid photopolymer resists for visible-laser imaging systems such as visible-laser-printing plates and circuit broads [6, 7]. Though we reported BTTBs in a PMMA film in the earlier investigation, there were both a main-staticquenching process from a singlet excited pyrroemethene dyes to a ground state of BTTB and a minor-dynamic quenching [6-i]. The sensitization mechanism of solution containing the photoinitiators, which is under similar condition to that of liquid photopolymer resists, is not clear yet. In this paper we describe the substituent effect in sensitization processes of pyrromethene dyes in acetonitrile (Fig. 1).

\section{Expermentals}

EBP, HBP, PBP were prepared according to the literature procedure [8]. BTTB was purchased from NOF Corporation. Acetonitrile was spectrograde and purchased from Wako Pure Chemical Industries, Ltd.

UV-VIS and fluorescence spectra were recorded with a Hiatchi U-3000 spectrophotometer and a Hitachi F- 454500 fluorescence spectrometer, respectively. Fluorescence decays were measured by a time resolved fluorescence spectrometer Horiba Corp. NAES-550 (single photon counting). All samples solutions were degassed with nitrogen for $20 \mathrm{~min}$. before the measurement. 
<smiles></smiles>

EBP: $R_{1}=-\mathrm{Me}, \quad \mathrm{R}_{2}=-\mathrm{Et}$ HBP: $R_{1}=-M e, \quad R_{2}=-H$ PBP: $R_{1}=-P h, \quad R_{2}=-E t$

\section{Laser light}

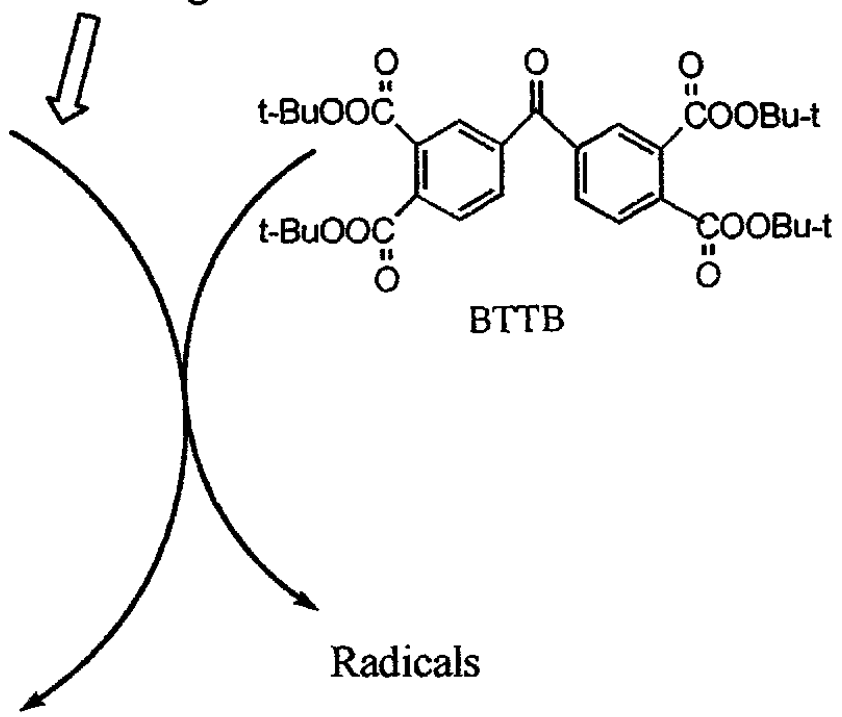

Acrylate monomers

Polymerization

Fig. 1. Photoinitiation systems.

Cyclic-voltammetric measurements were made on a Hokuto-Denko Function Generator HB-104 and Potentiostat HA-301 using a threeelectrode system. A platinum disk served as the working electrode, a platinum wire as the counter electrode and an $\mathrm{Ag} / \mathrm{AgNO}_{3}(0.1 \mathrm{M})$ electrode as the reference electrode. An electrolyte solution of $0.1 \mathrm{M}$ tetra-nbutylammonium perchlorate (TBAP) in acetonitrile was used to measure the redox potentials of the dyes. All sample solutions were degassed with nitrogen for $10 \mathrm{~min}$ before the experiment.

\section{Results and Discussions}

Fig. 2 shows absorption and fluorescence spectrum on $355 \mathrm{~nm}$ excitation for HBP in acetonitrile, and those for HBP and EBP in acetonitrile were described [9]. Absorption and fluorescence spectra for HBP, EBP and
PBP in acetonitrile have analogous profiles with absorption maximum at 491,514 , and $519 \mathrm{~nm}$, and fluorescence maximum at 501 , 531 and $534 \mathrm{~nm}$ for HBP, EBP and PBP, respectively.

In the fluorescence spectra in acetonitrile excited at different wavelength, 334 and $420 \mathrm{~nm}$, no significant change on the fluorescence spectrum was observed for the dyes.

Single photon counting. The profile of the decay of the fluorescence for the dyes in the presence and absence of BTTB on the excitation of 5 ns pulse were analyzed by means of single photon counting.

Fig. 3 shows the logarithmic plots of fluorescence decay in the absence and presence of BTTB. The logarithmic plots of fluorescence for the dyes reduced convexly over $10 \sim 20 \mathrm{~ns}$ and then reduced linearly over $20 \sim$ $50 \mathrm{~ns}$. The convex curve of the fluorescence 
decay was due to the effect of the excitationlamp, and was fittede to the simulation curve simulated by single-exponential-fluorescencedecay model. The life times were $6.5,7.3$ and $6.1 \mathrm{~ns}$ for HBP, EBP and PBP, respectively.

The life times of the dyes were decreased with the concentration of BTTB. The ratio $(\tau 0 / \tau)$ of the life times against the concentration of BTTB

exhibited a good linear relationship, which was fitted to stern-volmer plots, as depicted in Fig.4. From the slope of the plots, quenching rate constants (kq) were determined as 3.5, 3.0 and $4.3 \mathrm{~mol}^{-1} \mathrm{dm}^{3} \mathrm{~s}^{-1}$ for HBP, EBP and PBP. The fluorescence-quenching rate constants for the dyes were 2 orders of magnitude greater than triplet quenching-rate constant for TP.

In the sensitization mechanisms accompanied with an electron transfer

from photo-excited dyes to BTTB, the redox potentials of dyes were measured by cyclic voltammetry. The free energy change $(\triangle G)$ for electron transfer was obtained from Rhem and Weller equation [10] as $\triangle \mathrm{G}=-0.29,-0.21$ and $-0.22 \mathrm{eV}$ for HBP, EBP and PBP; $\triangle \mathrm{G}=$ Eox - Ered - Eoo - C, where the oxidation potential (Eox) is determined from the peak oxidation potential, Eox $=0.74,0.77$ and $0.76 \mathrm{~V}$ (vs. $\mathrm{Ag} / \mathrm{AgNO}_{3}$ ) for $\mathrm{HBP}, \mathrm{EBP}$ and $\mathrm{PBP}$, correspondng to Eox $=1.08,1.11$ and $1.10 \mathrm{~V}$ (vs. SCE) for HBP, EBP and PBP, Eoo is the excitation energy of the dye-singlet-excited state, Eoo $=2.45,2.40$ and 2.40

$\mathrm{eV}$ for HBP, EBP and PBP, Ered $=-1.18 \mathrm{~V}$ (SCE) for BTTB [11] and C, coulombic term, usually assumed to value ca. $0.1 \mathrm{eV}$ for acetonitrile solvent. The low free energy change $(\triangle G)$ indicates the possibility of an efficient-electron transfer from the singlet excited state of the dyes to the ground state of BTTB .

The molecular orbital calculations of 5phenyl pyrromethene- $\mathrm{BF}_{2}$ (PHP) using MINDO-AM1 and MNDO-psDI method were reported by $\mathrm{Li}$ et al. [12]. The ground state $\left.\mathrm{S}_{0}\right)$ of PHP has one stable conformer in which phenyl group is rotated $52^{\circ}$ against the rigorously planer pyrromethene framework. The photoexcited state $\left(S_{1}\right)$ of PHP has two stable conformers; one is a fluorescent metastable state with a phenyl ring rotated $37^{\circ}$, and the other is a nonfluorescent relaxed state with a non-twisted-phenyl ring against the plane

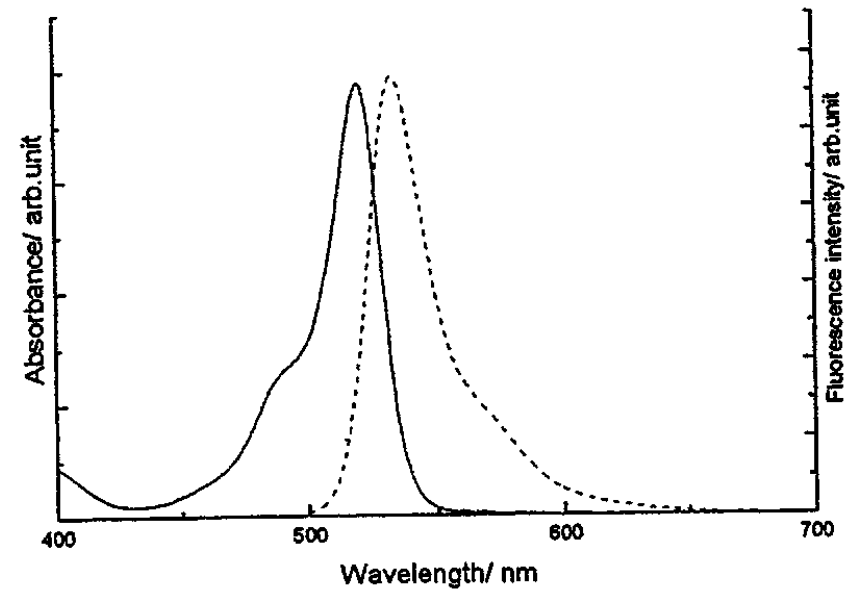

Fig. 2. Absorption (solid line) and fluorescence (dashed line) spectra of PBP in acetonitrile.

Substance concentrations were $7.63,6.28$ and $7.63 \times$ $10^{-6} \mathrm{~mol} \mathrm{dm}^{-3}$ for PBP ; excitation wavelength $355 \mathrm{~nm}$. Absorption coefficient $(\varepsilon)$ at maxmum ; $\varepsilon=5.1 \mathrm{x}$ $10^{4} \mathrm{~mol}^{-1} \mathrm{dm}^{3} \mathrm{~cm}^{-1}$ for PBP.

defined by atoms 4,5 , and 6 , and the pyrromethene framework significantly distorted $35^{\circ}$ out of plane due to the large resonance stabilization of phenyl ring and atoms 4,5 , and 6 , which has a significantly short lifetime of 15 ps.

Taking consideration into the bulkysubstituent effect in molecular orbital calculation for PHP, the relaxation mechanism of photoexcited EBP and PBP can be predicted as depicted in Fig. 6. The ground state has a stable conformer with fully rotated ethyl and phenyl group for EBP and PBP with respect to the rigorously planer pyrromethene

framework. The ground state is photoexcited to a singlet excited state $\left(S_{1}(A)\right)$ by absorbing a laser pulse. $S_{1}(A)$ deactivates accompanied with the large nucleous change. $S_{1}(A)$ for EBP undergoes rotation of ethyl groups to a relaxed state $\left(S_{1}(B)\right)$ with moderately rotated ethyl, and $S_{1}(A)$ for PBP undergoes rotation of phenyl group to a relaxed state $\left(S_{1}(B)\right)$ with moderatly rotated phenyl, subsequently going to a fullyrelaxed state such as a $\left(S_{1}(C)\right)$ with the lesstwisted phenyl ring due to the large resonace stabilization between phenyl group and dye chromophore. In case of HBP, the repulsion between substituents is lower than that for EBP and HBP. Thus the relation-energy gap from 

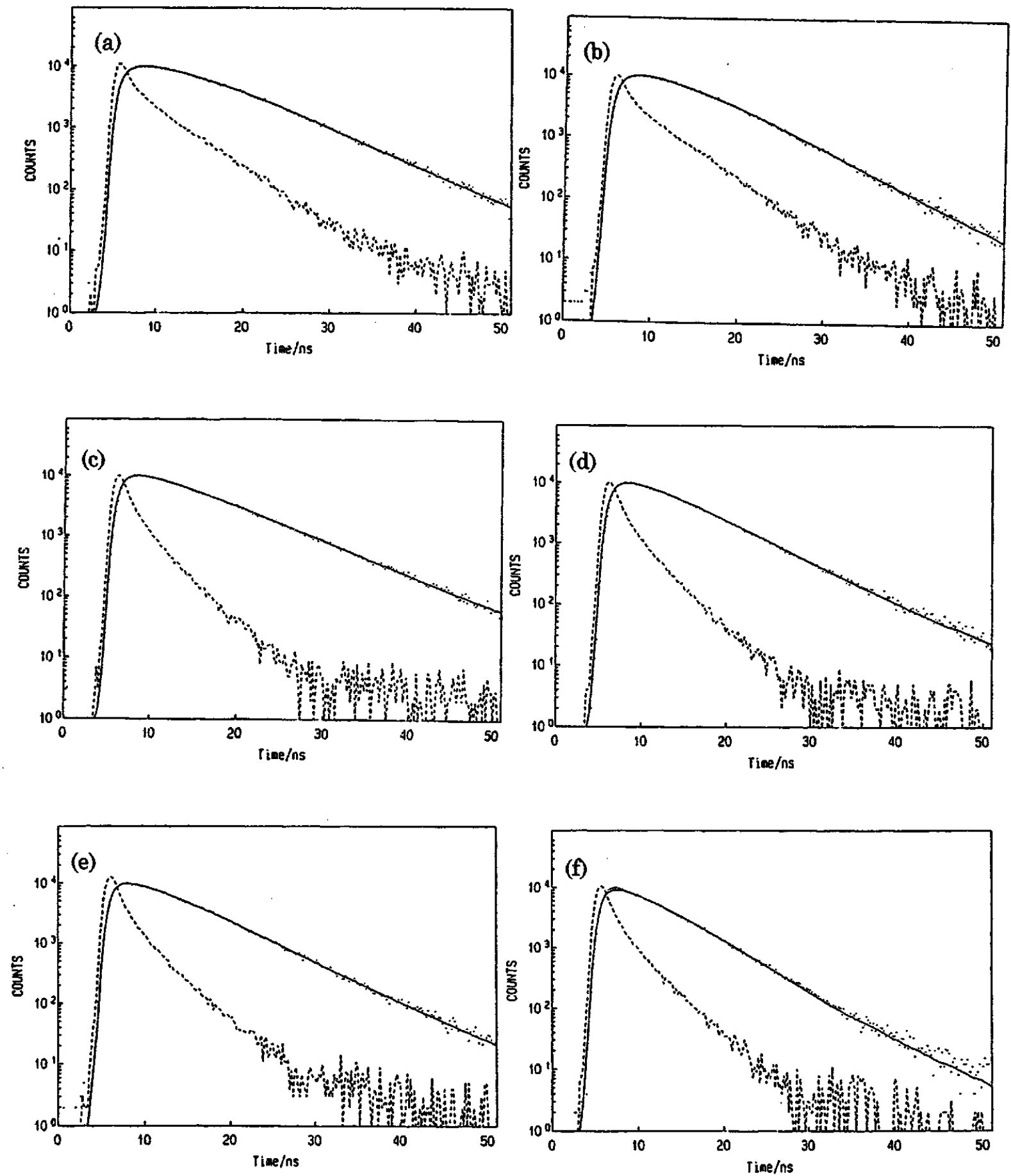

Fig. 3. Fluorescence decays of HBP (a), EBP (c), and PBP (e) in the absence of BTTB, and of HBP (b), EBP (d), and PBP ( $f$ ) in the absence of BTTB. Dashed line cure is logarithmic plots of lamp. Substance concentration $6.1 \times 10^{-6}, 1.3 \times 10^{-5}$, and $6.3 \times 10^{-6} \mathrm{~mol} \mathrm{dm}^{-3}$ for HBP, EBP and PBP, respectively and $10^{-2}$ $\mathrm{mol} \mathrm{dm}^{-3}$ for BTTB; detection wavelength more than $550 \mathrm{~nm}$ using cut pass filter (Toshiba Electric Corp. Glass filter O-56); excitation wavelength of pulse lamp $\left(\mathrm{I}_{1}\right), 490,515$, and $520 \mathrm{~nm}$ for HBP, EBP, and PBP, 

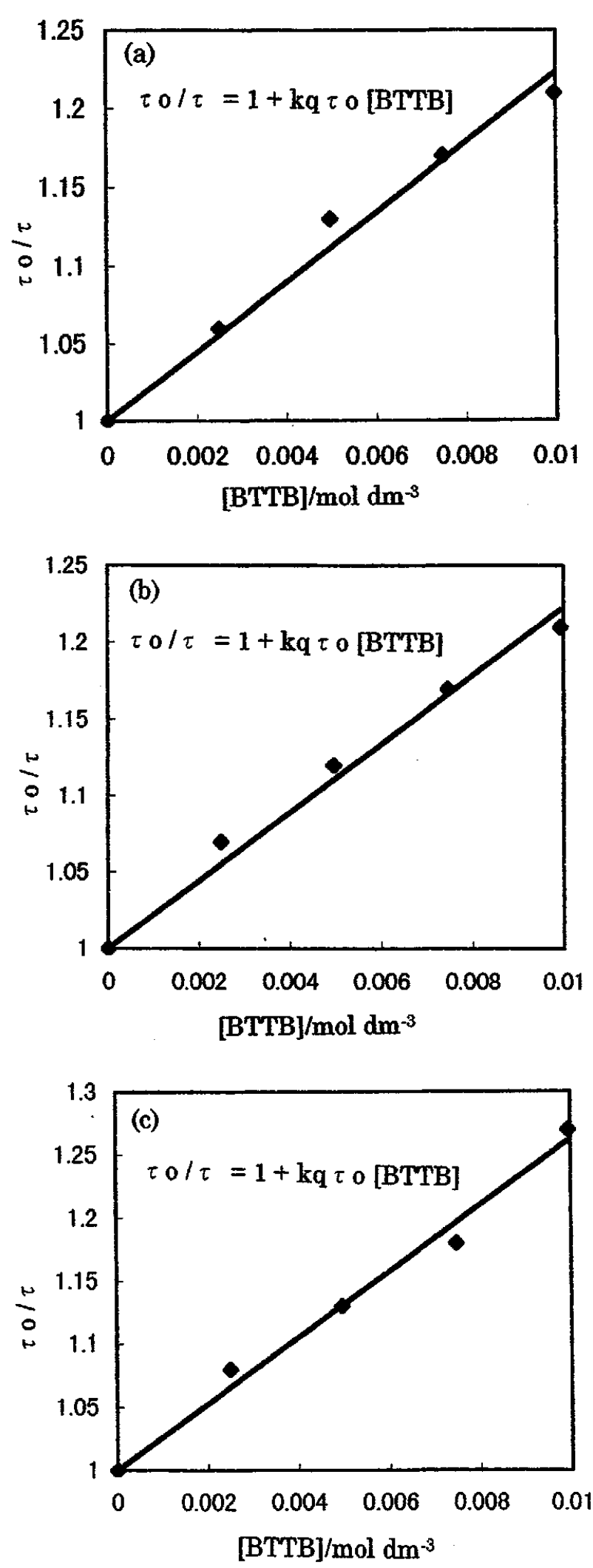

Fig. 4. Ratio $(\tau \circ / \tau)$ of tluorescence life time for HBP (a), EBP (b), and PBP (c) against BTTB (concentration).

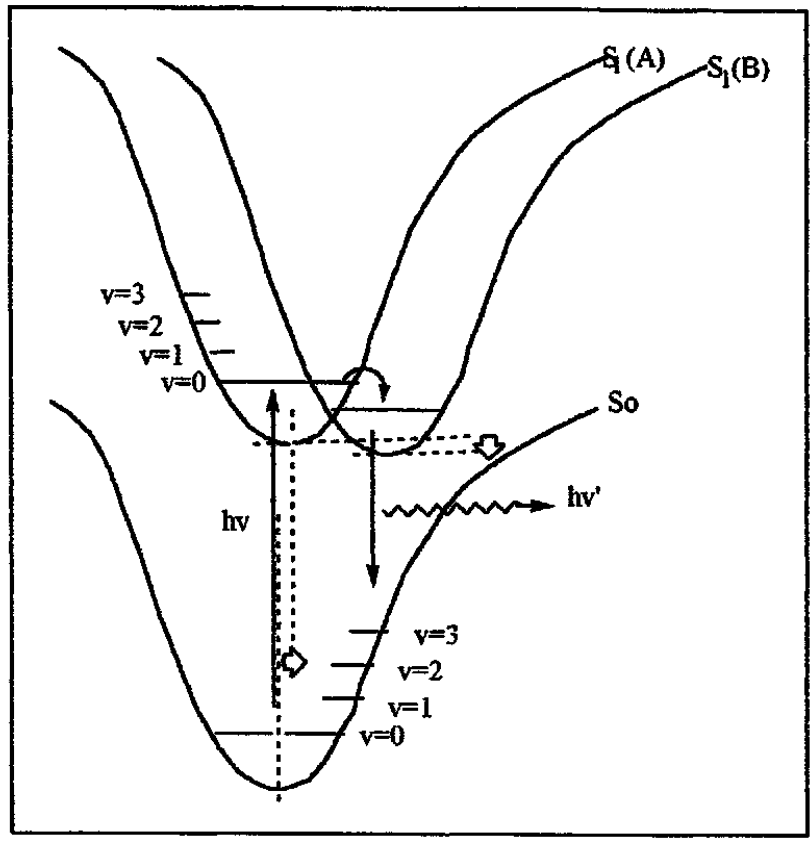

Fig. 5. Potential energy vs. nuclear configuration.

$S_{1}(A)$ to $S_{1}(B)$ is lower, and there is no state such as a $S_{1}(C)$ due to no resonance stabilization such as phenyl ring. $S_{1}(B)$ for the dyes decays radiatively or nonradiatively, or is quenched by BTTB.

The repulsion energy between phenyl ring and methyl groups, or between ethyl and methyl groups should increase the potential energy of the ground state, shifting absorption and fluorescence maximum to red, and the rotation of the bulky substituents in relaxation from $S_{1}(A)$ to $S_{1}(B)$ induces large-nuclearconfiguration change. Thus the longer absorption maximum for EBP and PBP than that for HBP, and the larger stokes shift for EBP and PBP than that for HBP support the large effect of the bulky substituents (Figure 2). The absorption maximum in $490-520 \mathrm{~nm}$ of the dyes are useful for laser imaging system equipped with $532 \mathrm{~nm}$ FD-Yag laser, and especially the absorption maximum at 514 and $519 \mathrm{~nm}$ for EBP and PBP is fitted to the emission light wavelength of FD-YAG laser.

The quenching-rate constant for PBP is larger than that for HBP and EBP. The reason of the large rate constant for PBP is not clear yet. However, it is considered that the spread of chromophore in PBP by phenyl ring increased the probability of electron transfer from the dye to BTTB.

Generally, liquid photoresists are used under air atmosphere. Since triplet lifetimes of 
general dyes under nitrogen atmosphere, $10^{-6}$ $10^{-1} \mathrm{~s}$ [13], and are efficiently reduced to $1-10 \mathrm{~ns}$ by oxygen in solutions as assuming the quenching rate of triplets by oxygen; $10^{11} \mathrm{~mol}^{-1}$ $\mathrm{dm}^{3} \mathrm{~s}^{-1}$ and the concentration of oxygen pressure in solutions at one atmopsphere of air; $0.03 \mathrm{~mol} \mathrm{dm}^{-3}$ [13], the lifetime of TP triplet under air atmosphere shuld be same order of magnitude as the fluorescence lifetime for the pyrromethene dyes. The highly efficient fluorescent character for pyrromethene dyes, and the two order of magnitude higher quenching rate constant of fluorescence for pyrromethene dyes by BTTB than the triplet quenching quenching rate constant for TP by BTTB implies the efficient sensitization character of the pyrromethene dyes for liquid photoresists.

Finally it is suggested that the pyrromethene dyes have high performance as a sensitization dye for liquid photoresist due to the high quenching rate constant, and the absorption maximum fitted to argon ion laser or FD-YAG laser.

\section{References}

1. F. Morlet-Savay, J. P. Fouassier,T. Matsumoto and K. Inomata, Polym. Adv. Technol., 5, 56 (1994).

2. G. Sathyamoorthi, J. H. Boyer, T. H. Allik, and S. Chandra, Heteroatom Chemistry, 5, 403 (1994).

3. a) J. H. Boyer, A. Haag, M. L.Soong, K. Thangaraj, T. G. Pavlopoulos, Appl. Opt. 30 , 3788 (1994), b) T. V. Pavlopoulos, J. H. Boyer, SPIE Visble and UV Laser, 2115, 231 (1994), c) T. G. Pavlopoulos, . H. Boyer, M. Shah, K. Thangaraj, M. L. Soong, Appl. Opt. 29, 3885 (1990),

4. A. A. Gorman, I. Hamblett, T. A. King, M. D. Rahn, J. Photochem. Photobiol., A, 130, 127 (2000).

5. a) J. L. Seifert, R.E. Connor, S. A. Kushon, M. Wang, B. A. Armitage, J. Am. Chem. Soc., 121, 2987 (1999), b) S. C. Hung, J. Y. Ju, R. A. Mathies, A. N. Glaser, Anual. Biochem. , 243, 15 (1996).

6. a) T. Urano, H. Nagasaka, N. Tsuchiyama and S. Shimizu, Mitsubishi Kasei $R$ and D, 2, 73 (1991); b) H. Nagasaka, T. Urano and M.
Tsuchiyama, Mitsubishi Kasei $R$ and $D ; 1,52$ $(1992) ; \quad c)$ T. Urano, K. O. Nguyen, H. Nagasaka, M. Tsuchiya, S. Shimizu, H. Itoh and Y. Senda, Bull. Chem. Soc. Jpn., 67, 1074 (1994); d) T. Urano, H. Nagasaka, M. Tsuchiyama, S. Shimizu, K. Kawazoe, M. Shimizu and T. Yamaoka, Bull. Chen. Soc. Jpn.; 68, 1661 (1995); e) T. Urano, H. Nagasaka, S. Shimizu, H. Ito, M. Shimizu, S. Takahara and T. Yamaoka, Bull. Chem. Soc. Jpon., 69, 693 (1996); f) H. Itoh, A. Takada, H. Kudo, H. Yokoyama, Y. Senda, T. Urano and $H$. Nagasaka, Bull. Chem. Soc. Jpn, 70, 2221 (1997); g) T. Urano, H. Nagasaka, S. Shimizu, H. Itoh, M. Shimizu, S. Takahara and T. Yamaoka, Bull. Chem. Soc. Jpn., 70, 1659 (1997); h) T. Urano, R. Takasaki, S. Ikeda and H. Itoh, Imag. Sci. J., 46, 6 (1998); i) T. Urano, H. Iton and T. Yamaoka, Polym. Adv. Technol., 10, 1 (1999) ; j) T. Urano, E. Ohno-Okumura, K. Sakamoto and H. Ito, J. Photopolym. Sci. Technol., 12 , 747 (1999).

7. T. Yamaoka, Y. Zhang and K. Koseki, $J$. Appl. Polymer. Sci., 38, 1271 (1989).

8. M. Shah, K. Thangaraj, M. L. Soong, L. T. Wolford, J. H. Boyer, I. R. Politer and T. G. Pavlopoulos, Heteroatom Chemistry, 1, 389 (1990).

9. T. urano, E.Ohno-Okumura, K. Sakamoto, S. Suzuki and T. Yamaoka, J. Phopolym. Sci. Technol., under publishing.

10 . a) D. Rhem and A. Weller, Isr. J. Chem., 259 (1970); b) D. Rhem and A. Weller, Ber. Bunsenges. Phys. Chem., 72, 2185 (1962); c) F.Scandola and V. Balzani, J. Am. Chem. Soc., 101, 6140 (1979).

11. Y. Goto, E. Yamada, M. Nakayama, K. Tokumaru and T. Arai, Nippon Kagaku Kaisi , 1027 (1987).

12. F. Li, S. I. Yang, Y. Ciringh, J. Seth, C. H. Martin, III, D. L. Singh, D. Kim, R. R. Birge, D. F. Bocian, D. Holtern and J. S. Lindsey, J. Am. Chem. Soc., 120, 10001 (1998).

13. N.J. Turro, "Modern Molecular Photochenmistry," The Benjamin/

Cumming Company, Inc., Canada (1978) p. $117,179,354$. 